\title{
Analysis on the Comprehensive Production Capacity of Garden Ginseng Industry in Jilin Province
}

\author{
Guofeng Zhang \\ Department of Chinese Traditional Medicine Science, Jilin Agricultural Science and Technology College, \\ Jilin 132101, China \\ Email:578174945@qq.com
}

Keywords: Ginseng, Garden Ginseng, Comprehensive Productivity

\begin{abstract}
Jilin Province is the main producing area of ginseng. The ginseng industry is a dominant industry and pillar industry in Jilin Province and an important part of the economic development of Jilin Province. Based on the development of the garden ginseng industry from the 2007-2016 Jilin Statistical Yearbook in the past 10 years, this paper analyzes the status of the comprehensive production capacity of the ginseng industry in Jilin Province from the indicators of planting area, total output and yield, and strives to revitalize the ginseng of Jilin Province. The industry provides a reference for promoting local economic development.
\end{abstract}

\section{Introduction}

Ginseng is a treasure in the treasure house of Chinese herbal medicine resources, and has high medicinal value and economic value [1]. Jilin Province is a major province for ginseng planting, production and processing. It is the world's main ginseng production area. The province's ginseng planting area accounts for more than $80 \%$ of the country's total, and its output accounts for $80 \%$ of the national output, accounting for $70 \%$ of global production [2]. With the improvement of people's health awareness, the demand for ginseng will increase year by year, the price of ginseng will increase as the market demand increases, and the market prospect of ginseng will become better and better. This paper analyzes the industrial production capacity of the garden ginseng industry through the analysis of the ginseng industry in Jilin Province, and tries to offer advice and suggestions through the scientific research and the development of the ginseng industry in Jilin Province.

\section{Analysis of the Current Situation of Garden Ginseng Industry}

From the statistical data in Figure 1, between 2007 and 2013, the growth rate of planting area of garden ginseng in Jilin Province is relatively stable, with an average annual planting area of 2.92 $\mathrm{hm}^{2}$; from 2013, the planting area of garden ginseng is increasing, it reached an all-time high of $6.52 \mathrm{hm}^{2}$ in 2014, an increase of 96.9\% compared with 2013.From the change of the planting area of the garden ginseng in Figure 1, it may be caused by the following reasons. First, the industrial positioning has been affected by the international and domestic situation in recent years. Jilin Province pays special attention to the development of ginseng industry. The ginseng industry has become a dominant industry and provides opportunities for the development of ginseng industry in Jilin Province. Second, the demand orientation. With the enhancement of people's health awareness, ginseng is an important nourishing product with strong cultural heritage and long history. It is deeply loved by consumers, resulting in increasing demand and rising prices. Third, resources and technological advantages, Jilin Province is a large province of ginseng resources, especially the ginseng planting in non-forest land has made major breakthroughs in solving the problem of key cultivation techniques, and the vast farmland provides the material basis for garden ginseng farming. Fourth, the conditional advantage. There are many scientific research institutes in Jilin Province, including Jilin Agricultural University. More than 80 scientific research institutions and institutes have provided technical support for the development of modern ginseng industry. 


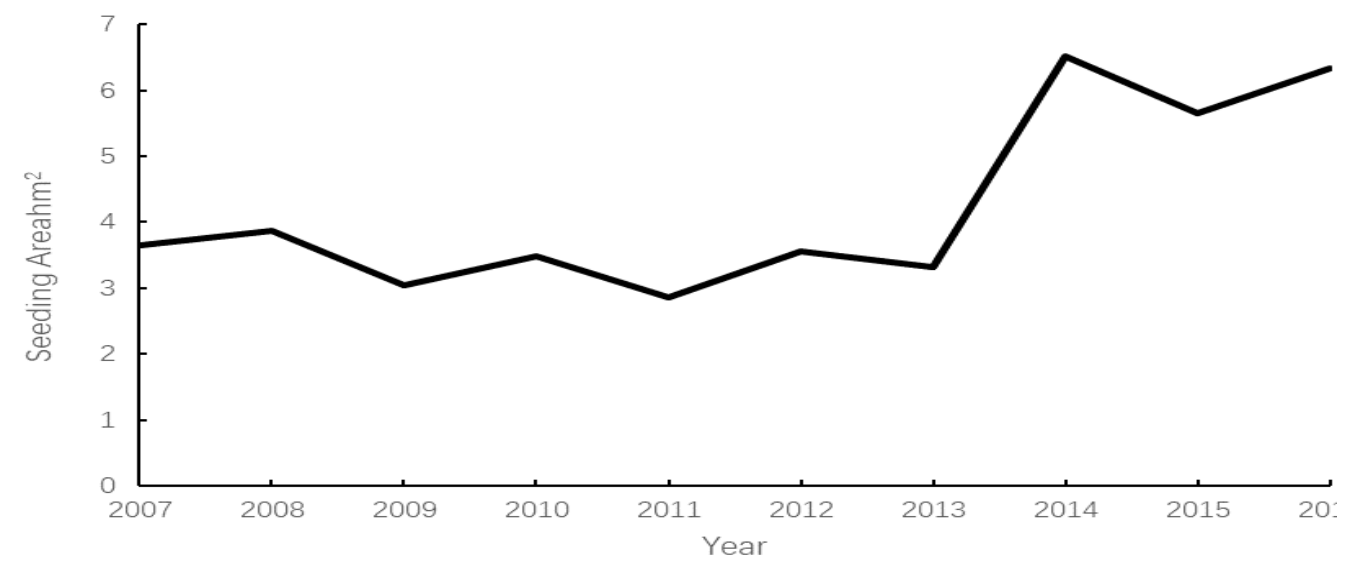

Fig.1. Changes in planting area of garden ginseng in Jilin Province from 2007 to 2016 (unit: hm²)

As can be seen from Figure 2, the total output of garden ginseng reached 307,500 tons in the past 10 years, and the total output was 30.75 million tons. The total output in 2011 reached 36,900 tons. Since 2014, the total output of garden ginseng has been declining, and the highest total output from garden ginseng has dropped to 27,000 tons, a decrease of $26.9 \%$, which is negatively correlated with the planting area of garden ginseng. It can be seen that due to the influence of national policies, traditional deforestation has been banned. The increase in planting area of garden ginseng is mainly based on farmland. Because ginseng has higher requirements on the growing environment, the farmland ginseng environment is compared with the original ecological forest. The gap is large, which has a certain impact on the yield and quality of garden ginseng. Eventually, the total yield of ginseng does not increase with the increase of planting area, but it will decline. The research on key technologies of non-forest land ginseng will become the key to restrict the development of ginseng industry.

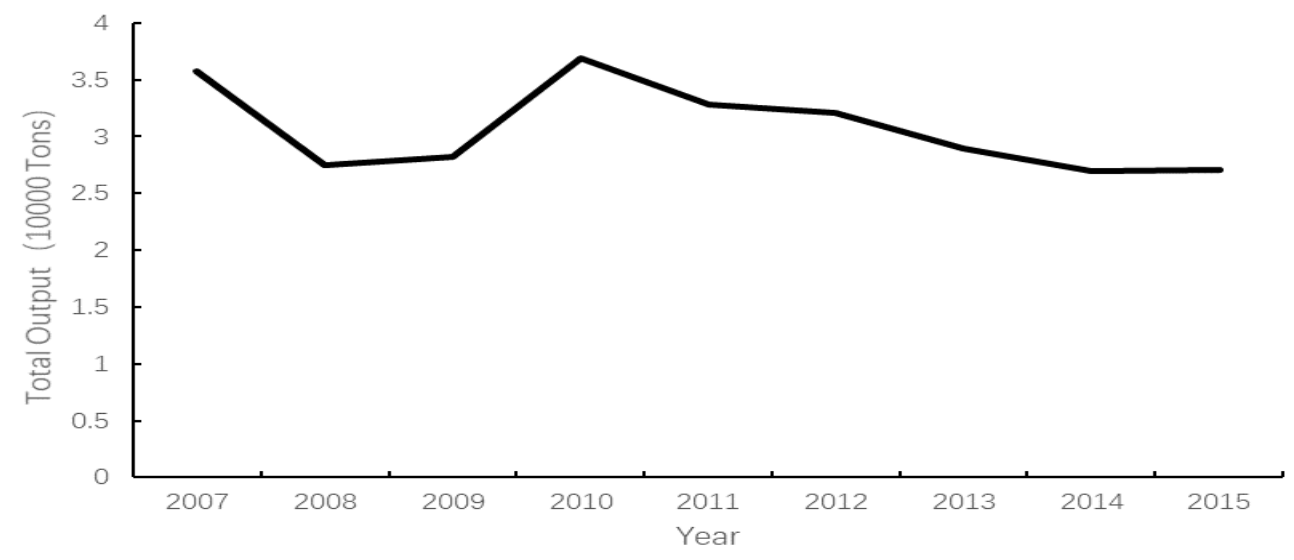

Fig.2. Changes in total production of garden ginseng in Jilin Province from 2007 to 2016 (unit: 10,000 tons)

It can be seen from Figure 3 that from 2007 to 2013, the yield of garden ginseng continued to increase. In 2011, the highest value of ginseng yield was $13113 \mathrm{~kg} / \mathrm{ha}$, which exceeded the 10,000 $\mathrm{kg} / \mathrm{ha}$ mark, which promoted the development of ginseng industry. However, since 2014, the yield of garden ginseng has been declining, nearly doubled compared with the previous year. In 2016, it reached the lowest point of 4,284.36 kg/ha in the past 10 years. It can be seen that the yield of garden ginseng is positively correlated with the total yield of garden ginseng, negatively correlated with the planting area of garden ginseng, and decreases with the decrease of total ginseng yield. 


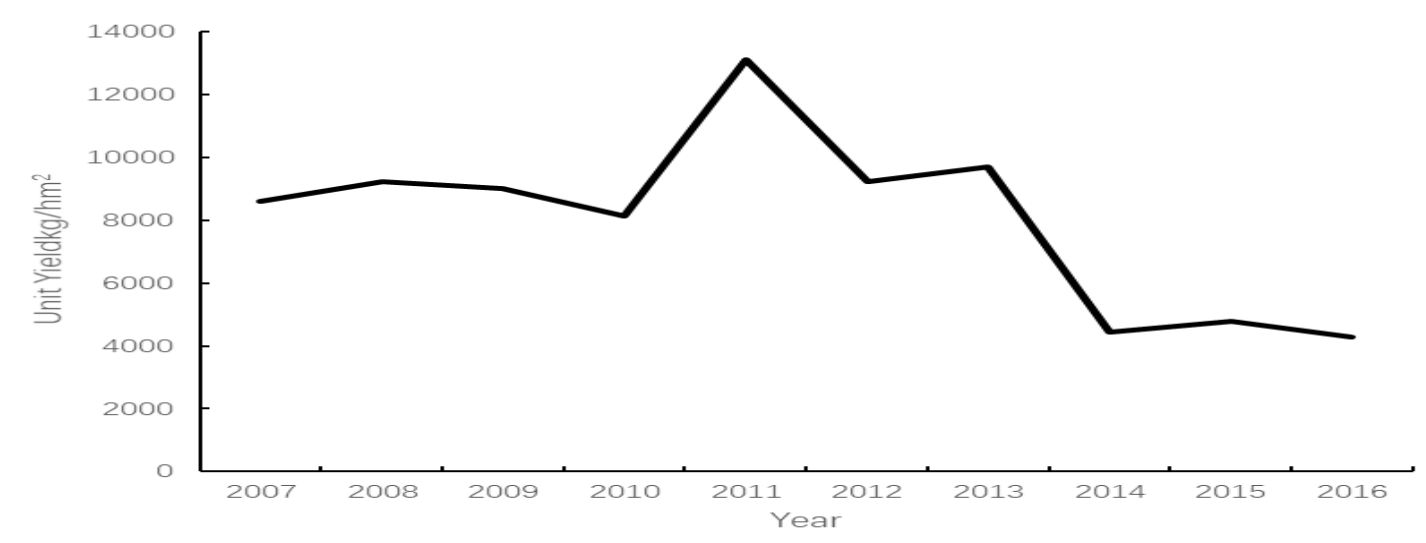

Fig.3. Changes in total ginseng production in Jilin Province from 2007 to 2013 (unit: kg/ha)

\section{The Countermeasures Suggestion}

For the long-term development of the garden ginseng industry, we must adhere to the consumer demand-oriented, increase the government's protection of the ginseng industry, integrate resources, create a good operating environment, formulate ginseng quality standards, and support industrial security measures. First, the government should step up guidance, promote universal health care, build a healthy culture, and create a public opinion atmosphere. Second, increase capital investment, especially $R \& D$ investment, master key core technologies, improve $R \& D$ capabilities and levels, and increase the demonstration and promotion of garden ginseng industry to create an integrated production and sales of ginseng industry. Third, strengthen cooperation, attract foreign investment on preferential terms, inject - foreign capital, and expand research and development of ginseng products. Fourth, provide market support conditions, severely crack down on unfair competition, illegal procurement, and sales behavior, and establish a sound and complete return system and purchase preferential system.

Jilin Province ginseng industry has a superior geographical position, and ginseng planting has a long history. It is the most important ginseng production area in the country. Compared with Korean ginseng, China has fewer ginseng brands and less international recognition. Ginseng branding is a key factor restricting the development of ginseng industry and an important measure to solve the sustainable development of ginseng industry. Through branded marketing, it can promote cultural communication, eliminate regional cultural differences and consumption habits, reach consensus, and form correct consumption values. At the same time, with the help of green concepts, we can establish an innovative ginseng industry intensive and efficient model to form the characteristics of Jilin Province ginseng brand and the implementation of branding, which is conducive to the formation of comprehensive advantages of ginseng industry and promotion of ginseng industry upgrading.

\section{Conclusion}

The ginseng industry is an important industry in Jilin Province. In the context of revitalizing the old industrial base in Jilin Province, solving the problem of "Ginseng industry development in Jilin Province” is not only an inevitable requirement for accelerating the economic development of Jilin Province, but also maintaining the sustained and rapid coordination of the national economy. The inevitable requirement of development is an inevitable requirement for realizing the grand goal of building a well-off society in an all-round way.

\section{Acknowledgements}

(1) Key Laboratory of Technology Innovation in Production and Utilization of Herbal Medicine of Jilin Provincial Highwa, 2017. (2) Chinese medicine school level key disciplines of Jilin 
Agricultural Science and Technology college Department of Chinese Taditional Medicine Science, 2018. (3) This study was supported by National Natural Science Foundation of China subsidization project study on insect resistance of ginsenosides and its effect on the evolution of environmental suitability of ginseng (No. 31470420). (4) Study on Soil Remediation Technology of conversion of ginseng-plant to Forestland in Eastern Mountainous Area of Jilin Province (No. Ji UNESCO combined Words2016209).

\section{References}

[1] Yuguang GAO and so on. Problems and countermeasures for the sustainable development of ginseng industry in Jilin Province [J]. Rural Science and Technology, 2017(35): 18-19.

[2] Yuxi LI and so on. Status quo of ginseng food industry development in Jilin Province [J]. Food Safety Guide, 2016 (27): 85. 